ISSN: 2638-5945

\title{
Lens Abces: A Case Report
}

\author{
Aymen Abdeljilil* \\ Ophthalmology Department B, HédiRais ophthalmology institute of Tunis, Tunisia
}

*Corresponding author: Aymen Abdeljilil, Ophthalmology Department B, HédiRais ophthalmology institute of Tunis, boulevard 9-Avril, 1006 Tunis, Tunisia

\section{Introduction}

Lesions of the lens are frequent during perforating trauma of the eye, in the majority of cases it is post traumatic cataract, however some cases of lens abscess have been reported in the literature and occur when a purulent infiltration develops inside the crystalline cortex [1].It is important to recognize this sequestered infection and to remove the abscess surgically to prevent chronic endophthalmitis.We report the case of an intra lenticular lens abscess treated surgically by phacoemulsification with good postoperative results.

\section{Clinical Case}

It is about a 53-year-old farmer patient, working as a farmer, with no significant medical history who consulted 15 days after trauma to the right eye with a plant thorn.The visual acuity of the right eye was limited to the counting of the fingers to 1 meter, the examination with the slit lamp found a conjunctival hyperemia with a perikeratic circle, a corneal central punctiform opacity which was not taking fluorescein; a tyndall in the anterior chamber; a total cataractand an arleflectic miosis with iridocrystalline synechiae over $360^{\circ}$. The examination with the fine slit finds a mass which bulges under the iris in the inferotemporal quadrant (figure 1), the fundus examination was inaccessible so we completed with an ultrasound exam which did not show any inflammation or abnormality in the vitreous or the retina.The visual acuity of the left eye was $10 / 10$ and the slit lamp examination was without abnormalities.In a first time we prescribed topic corticosteroids, hourly fortified antibiotic eye drops and cycloplegic, and intravenous large spectrum antibiotic and antifungal.The evolution at 15 days of treatment wasthe disappearance of hypopion, the reduction of inflammation and eye pain, with persistence of conjunctival hyperemia and sub iris mass.A presumptive diagnosis of intralenticular lens abscess was made, and a decision was made to drain the abscess surgically.

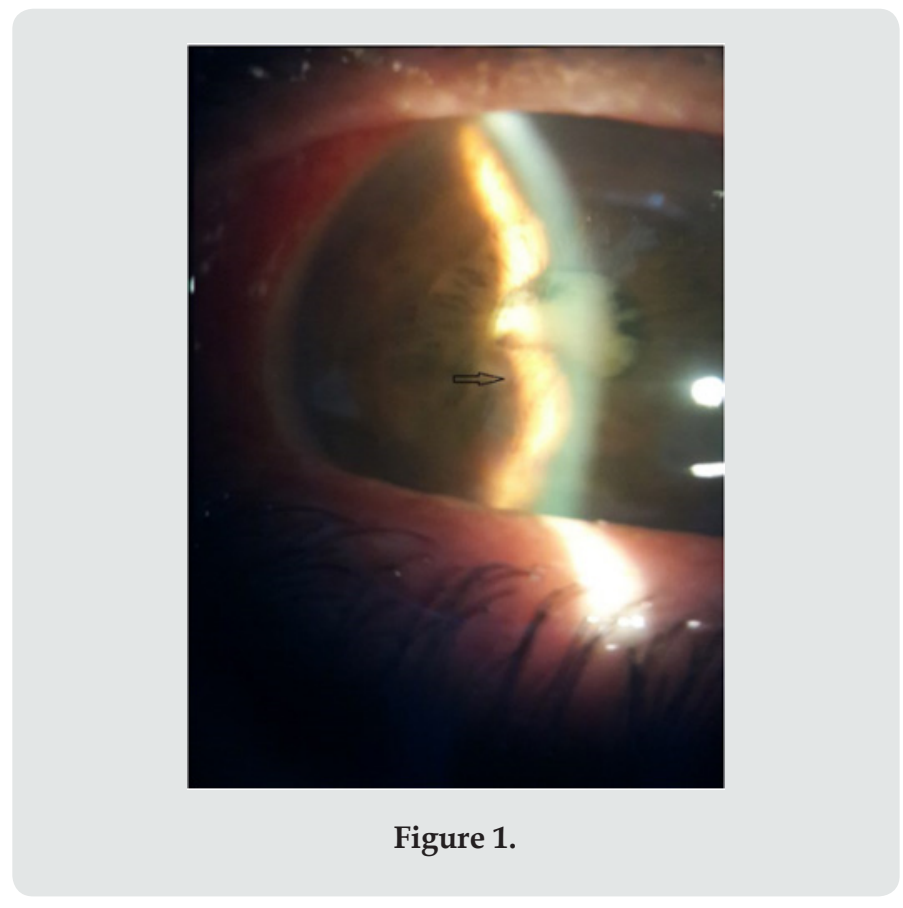

The patient was operated under local retro bulbar anaesthesia, the surgery was started by synechiolysis, and iris dilators were put in place revealing the abscess which was a homogeneous opacity, dense, yellowish white, well demarcated from the rest of the lens, with vascularization from the adhesion of the adjacent iris (Figure 2). This collection was punctuated and its purulent content was emptied into the anterior chamber (Figure 3); the pus has been removed and drained; and the remaining fibrous shell was detached from the iris and removed with forceps (Figure 4), phacoemulsification continued without incident and we ended with the placement of a lens implant in the capsular bag (Figure 5).The sequellar corneal opacity which presents the point of penetration of the thorn was better visualized after the dilation of the iris 
(Figure 6) Microbiological examination of pus, aqueous humor, and aspirated cortical material found Staphylococcus epidermidis which was sensitive to methicillin, so a topical and general antibiotic treatment was continued for 10 days, with clinical improvement and final visual acuity at $8 / 10$.

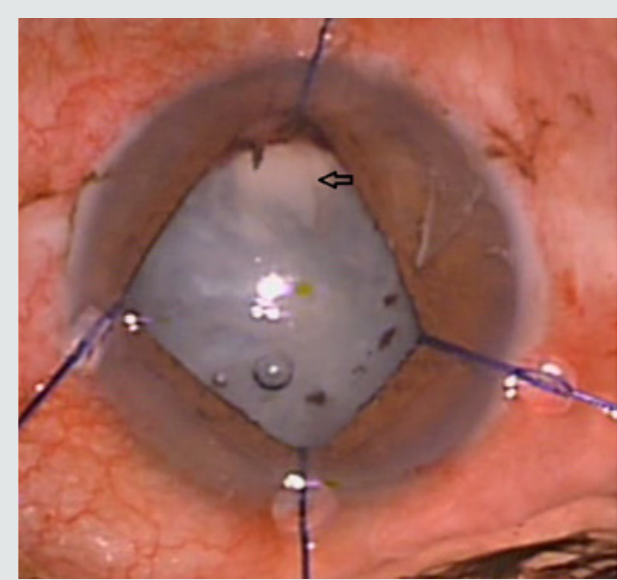

Figure 2.

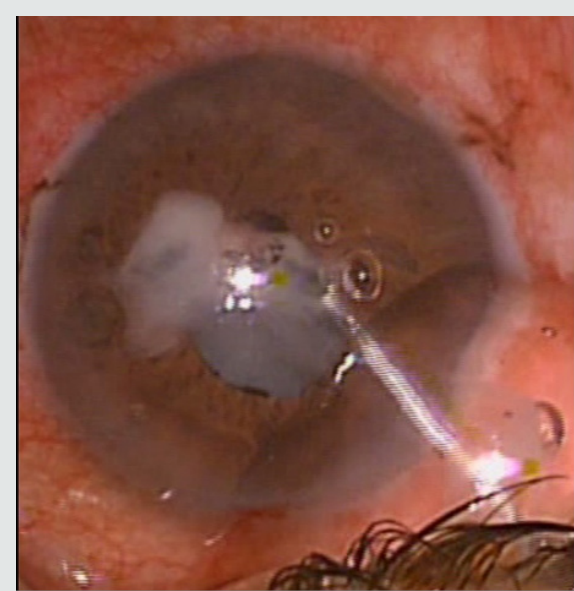

Figure 3.

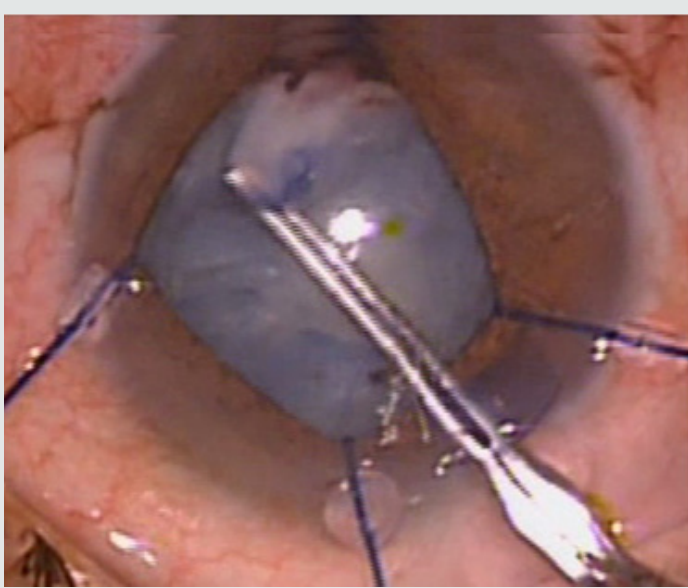

Figure 4.
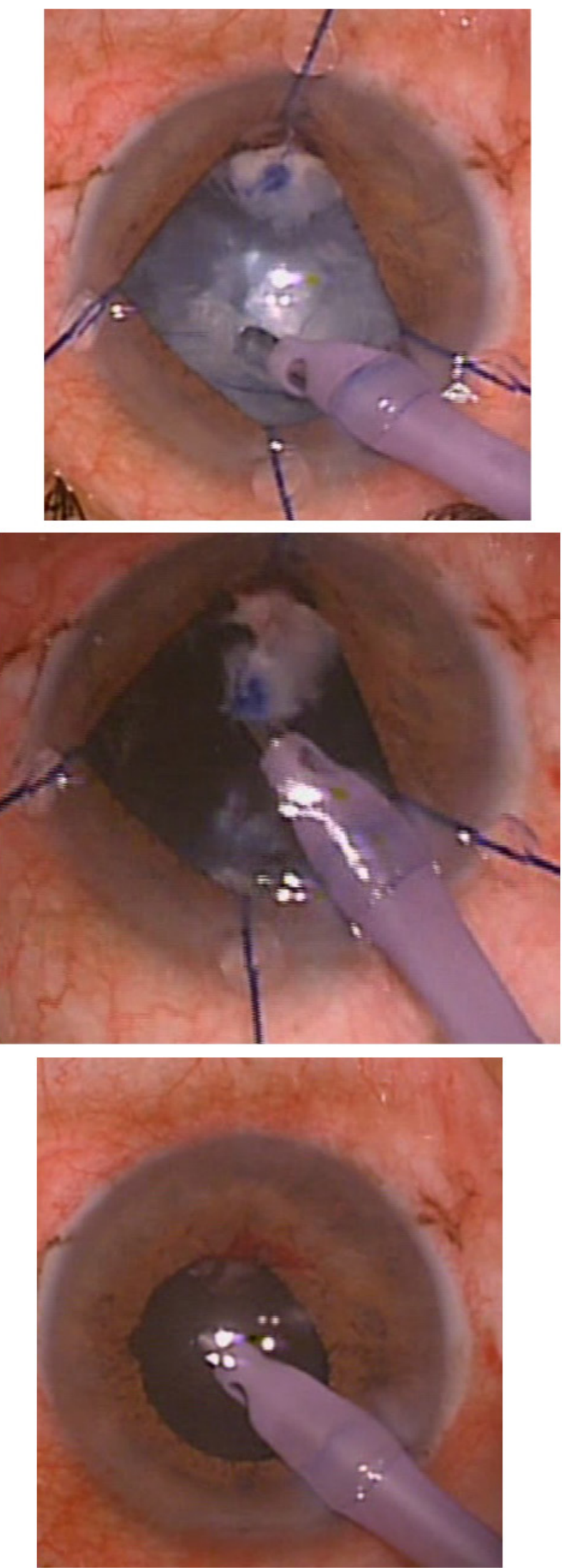

Figure 5. 


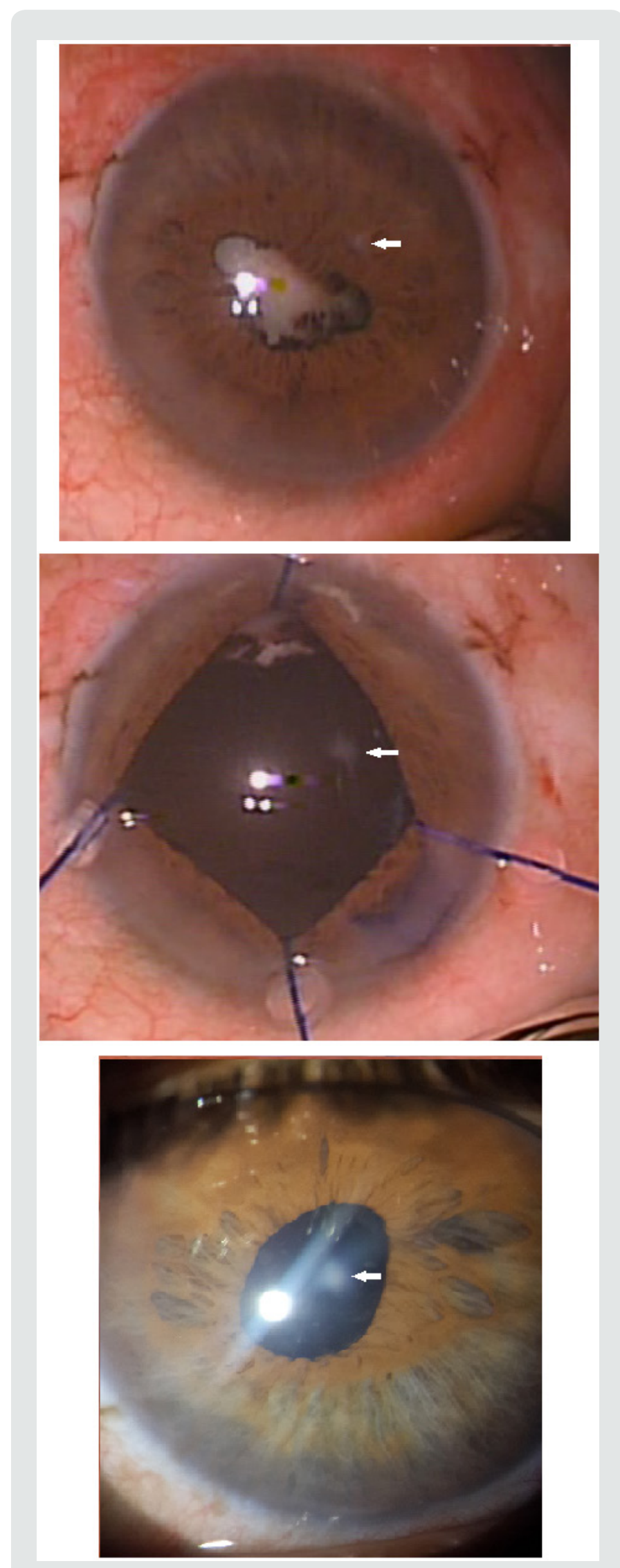

Figure 6.

\section{Discussion}

Lens abscesses are rare infections [2] that infiltrate the lenticular substance in the lens, these abscesses may occur after cataract surgery, metastatic infection, or trauma [3-5].Post traumatic lens abscesses are often caused by long, pointed and contaminated objects such as needles and thorns with direct inoculation into the lens cortex [2], this type of trauma is common in agricultural workers, as for our patient, the trauma was caused by a cactus thorn while working in the field.Although the lens is damaged in about $30 \%$ of the perforating lesions of the eyeball and $10 \%$ of all intraocular foreign bodies are found in the lens, lens lenticular abscesses are exceptional [6], this is probably due to protective effect of the anterior lens capsule.Busacca in its experimental studies has proven that the crystalline cortex constitutes an excellent place of culture for the germs and that the infection of the lens is only possible if the anterior or posterior capsule is perforated [1], if this capsular rupture is less than 2 millimeters in length, this can heal with the proliferation of epithelial cells in the lens. Indeed a certain number of reported cases in which intralenticular foreign bodies have remained in the lens for decades with little reaction or cataract formation [6]The lens capsule not only constitutes a barrier against infectious agents but also against the penetration of antibiotics and antifungals into the lens [2-7], thus surgical drainage of this sequestered infection is necessary after control of the initial inflammation [7]The majority of authors report a latency time between the trauma and the consultation for lens abscess ranging from a few weeks to a few months [1-8], this latency was 2 weeks for our patient.The most frequent causative agent is staphylococcus epidermidis [2,8], other microorganisms have been reported in the literature such as pseudomonas [2], pneumococcus [1], Propionibacterium acnes [6] as well as type of aspergillus [2], but in some cases no causative agent has been individualized [4] or else individualized only on a single type of sample like D'mellow, et al[8] who found a positive culture from of the crystalline cortex when it was negative from the aqueous humor and the histological examination of the iris showed only non-specific mononuclear infiltration.These results highlight the importance of multiplying the number and sites of samples taken during lens abscesses: from aqueous humor, pus and drained fibrin, and especially emulsified lens material where germs may not be accessible to the effect of antibiotics to ensure the maximum chance of individualizing a germ, especially since these samples are generally taken after a long antibiotic treatment [8].To treat this infection all the authors agree on the need to surgically drain the abscess and excise all infected or suspect tissues [2-9], but the procedure differs from one surgeon to another, some authors propose a browsing of the lens by the pars plana associated with a vitrectomy $[4,9]$, others propose when the posterior capsule is intact an extracapsular extraction of the lens without vitrectomy and the implantation of the lens during a second operation [8]. While other authors propose the placement of a lens implant during the same 
operation, especially when the infection has been well controlled before surgery [7].In our case we proceeded by a phacemulsification with implantation in the capsular bag of a lens implant with a good postoperative evolution without recurrence of the infection and a final visual acuity at 8/10.The prognosis for lenticular abscesses has literally changed; these infections previously reported as blinding in the first descriptions [1] can nowadays be perfectly treated $[2,7,8]$ thanks to the development of anti-infectious agents and especially to eye surgery, thus phacoemulsification allows at the same time drain the abscess and treat an associated post-traumatic cataract.

\section{Conclusion}

Post traumatic intra lenticular abscesses are rare infections, which are characterized by a specific traumatic mechanism by the direct inoculation of infectious agents inside the lens, and by a lag time between the trauma and the development of the infection, the management of these abscesses requires surgical drainage of the sequestered infection in addition to conventional anti-infectious treatments

\section{References}

1. Rychener RO, Ellett EC (1942) Abscess of the Crystalline Lens. American Journal of Ophthalmology 40: 285-292.
2. Rajaraman R, Lalitha P, Raghavan A, Palanisamy M, Prajna NV, et al. (2007) Traumatic lenticular abscess: clinical description and outcome. Am J Ophthalmol 144(1): 144-146.

3. Fish LA, Ragen MT, Smith RE, Lean J (1988) Propionibacterium acnes lens abscess after traumatic implantation of intralenticular cilia. Am J Ophthalmol 105(4): 423-424.

4. Kaushik S, Ram J, Dogra MR, Narang S, Brar GS, et al. (2001) Traumatic lens abscess with chornic endophthalmitis successfully treated with pars plana lensectomy and vitrectomy. Ophthalmic Surg Lasers 32(3): 239-242.

5. Liesegang TJ (1983) Traumatic lens abscess: report of a case. Ann Ophthalmol 15(12): 1151-1154.

6. Moore DC (1989) Intralenticular abscess. Aust N Z J Ophthalmol 17(3): 313-315.

7. Trpin S, Gracner T, Pahor D, Gracner B (2006) Phacoemulsification in isolated endogenous Candida albicans anterior uveitis with lens abscess in an intravenous methadone user. J Cataract Refract Surg 32(9): 15811583.

8. D'mellow G, Hirst LW, Whitby M, Nimmo G, Stallard K, et al. (1991) Intralenticular Infections. Ophthalmology 98(9): 1376-1378.

9. Shah GK, Vander J, Eagle RC (2000) Intralenticular Candida species abscess in a premature infant. Am J Ophthalmol 129(3): 390-391.

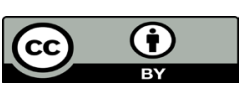

This work is licensed under Creative Commons Attribution 4.0 License

To Submit Your Article Click Here:

Submit Article

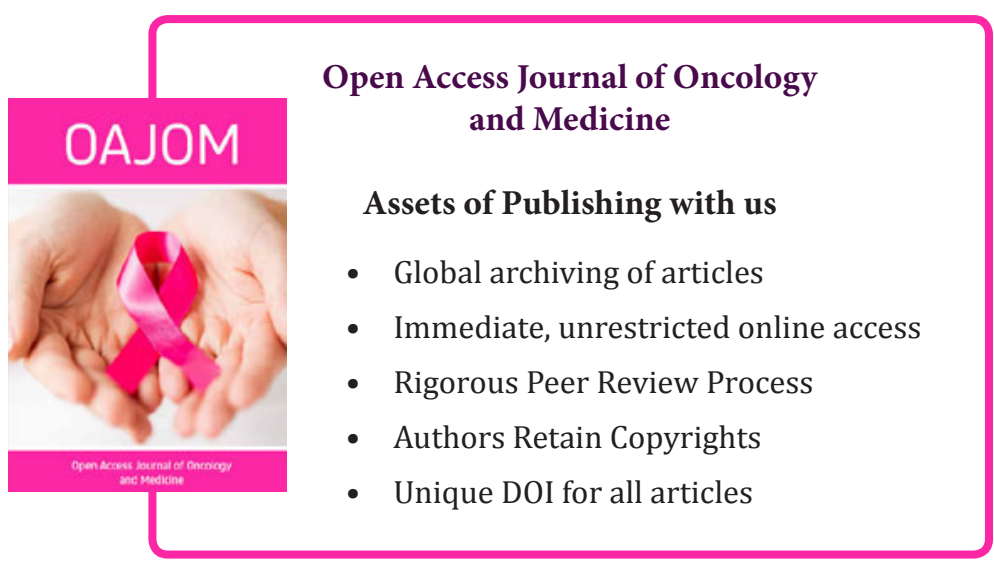

